\title{
POSTERIOR REVERSIBLE ENCEPHALOPATHY SYNDROME (PRES)- CASE REPORT \& REVIEW OF LITERATURE
}

\author{
Sarojini Raman'1, Chinmay Kumar Behera², Urmila Senapati ${ }^{3}$
}

${ }_{1}^{1}$ Associate Professor, Department of Pathology, Kalinga Institute of Medical Sciences, Bhubaneswar, Odisha, India.

${ }^{2}$ Associate Professor, Department of Paediatrics, Kalinga Institute of Medical Sciences \& PBMH, Bhubaneswar, Odisha, India.

3 Professor and HOD, Department of Pathology, Kalinga Institute of Medical Sciences, Bhubaneswar, Odisha, India.

HOW TO CITE THIS ARTICLE: Raman S, Behera CK, Senapati U. Posterior reversible encephalopathy syndrome (PRES)- case report \& review of literature. J. Evolution Med. Dent. Sci. 2019;8(24):1962-1965, DOI: 10.14260/jemds/2019/431

\section{PRESENTATION OF CASE}

A seven- year- old male child presented with generalised progressive swelling all over body with decreased urine output and nephrotic range proteinuria. He had history of headache \& visual impairment since 6 months. No history of fever, arthralgia, rashes or any other symptoms present. His blood pressure was 160/90 mmHg. Investigations: Hb- 6.37 gm/dl, TLC- 20,110/cmm, TPC- 4.1 lakh/cmm, Sr. Urea- 63 mg/dl, Sr. Creatinine- $1.1 \mathrm{mg} / \mathrm{dl}$, Sr. Total Protein- 3.7 gm/dl, Serum albumin- $1.4 \mathrm{gm} / \mathrm{dl}$, Total cholesterol- $351 \mathrm{mg} / \mathrm{dl}$, ANA1+Positive, ASO- Negative, Sickling test- Negative, Sr. C3- 78 $\mathrm{mg} / \mathrm{dl}, \mathrm{HIV} / \mathrm{HBs} A g-$ Negative, Urine RE/ME- Albumin 4+, pus cells- 0- 2/HPF, RBCs- 4- 6/HPF, hyaline cast- 0- 2/LPF, Spot urine protein- $814.8 \mathrm{mg} / \mathrm{dl}$, spot urine creatinine- 32.15 $\mathrm{mg} / \mathrm{dl}$, Urine protein creatinine ratio- $25.343 \mathrm{mg} / \mathrm{gm}$, Stool RE/ME- Strongyloides stercoralis, USG Abdomen \&pelvis showed cholelithiasis, ascites. MRI brain revealed bilateral occipital cortex cortical atrophy. Clinical diagnosis was Nephrotic syndrome associated PRES. Renal percutaneous biopsy was done. Histopathology showed presence of fetal glomeruli \& mild mesangial matrix accentuation in one glomerulus. (Fig. 1, 2) Features of acute tubular injury with atrophic tubules seen with moderate chronic inflammatory cell infiltration in interstitium. (Fig. 3) Blood vessels were unremarkable. Immunofluorescence study showed minimal positivity for IgM\& C3 antisera. So, the diagnosis of tubulointerstitial nephritis with mild mesangial sclerosis in association with PRES was given.

\section{DIFFERENTIAL DIAGNOSIS}

The non- specific clinical manifestations and multiplicity of radiological patterns increases the diagnostic challenges as various other conditions mimic PRES. 12

1. Acute Cerebral Ischaemia:

Unilateral lesions with a decreased diffusion coefficient.

2. Cerebral Venous Thrombosis:

Asymmetric, diffuse lesions.

3. Transient Cerebral Hyperaemia:

Develops after an episode of seizure or end arterectomy.

'Financial or Other Competing Interest': None.

Submission 09- 03-2019, Peer Review 17-04-2019,

Acceptance 23- 04- 2019, Published 17-06- 2019.

Corresponding Author:

Sarojini Raman,

Department of Pathology,

KIMS, Campus- 5, KIIT Campus,

Bhubaneswar-751024,Odisha, India.

E- mail:sraman10371@gmail.com

DOI: $10.14260 /$ jemds $/ 2019 / 431$

\section{(c) $(1) \ominus$}

4. Cerebral Oedema After Prolonged Hypoxaemia: Diffuse lesions with raised diffusion coefficient.

5. Infectious Encephalitis:

Shows increased cellularity of CSF, positive CSF Gram stain/ culture/ serology/PCR, associated with fever \& leukocytosis, may have unilateral affection in brain imaging studies.

6. Encephalitis Due to Other Causes:

History of malignancy/viral infection/vaccination present, positive detection of specific antibody in serum or CSF, imaging shows asymmetric unilateral or bilateral involvement.

7. Malignancy (Primary or Metastatic):

Insidious onset, history of weight loss, previously diagnosed primary tumour in other body parts, abnormal CSF cytology study, no clinical or radiological resolution, can be unilateral in brain imaging.

8. CNS Vasculitis:

Usually subacute in nature, increased CSF cellularity, oedema may be non- PRES- like.

9. Osmotic Demyelination Syndrome:

Previous treatment history of rapid normalisation of sodium or glucose concentrations present. It may affect areas other than parieto- occipital lobes, typical central pontine signal abnormality in a bat- wing shape in radiology.

10. Toxic Leukoencephalopathy:

Positive history of illicit drug use, affirmative drug or toxin screen, Magnetic resonance spectroscopy can show raised lactate and decreased $\mathrm{N}$ - acetyl aspartate concentrations in brain. 3,24

\section{PATHOLOGICAL DISCUSSION}

Posterior reversible encephalopathy syndrome (PRES) is a clinical syndrome with acute neurological deficits seen in association with autoimmune disorders, sepsis, renal failure, organ transplantation \& use of cytotoxic drugs. First coined by Hinchey in 1996, this entity is now being detected frequently due to advent of technology.

The exact pathophysiology of PRES is unclear, though theory of hyper- perfusion \& ischemia as the culprits are widely accepted. ${ }^{1}$ The true incidence and prevalence in children is unknown. ${ }^{2}$ As various body parts are simultaneously affected by this disorder experts from different stream may come across such cases like neurologists, nephrologists, oncologists, haematologists, intensivists and transplantation surgeons. ${ }^{3}$

The pathophysiologic mechanism of PRES remains an enigma. There are two contradicting hypotheses. As per the first theory when severe systemic hypertension overcomes the auto- regulatory capability of the cerebral vasculature, it leads to enhanced perfusion, arteriolar dilatation \& 
consequent injury to the capillaries. This results in extravasation of fluid, cells \& macromolecules producing vasogenic oedema. The oedema is initially localised to cortex \& later percolates into the subcortical white matter. The predilection for posterior segment of brain may be associated with lack of sympathetic innervation. The degree of hypertension is not always proportionate to the extent of vasogenic oedema in many cases \& PRES may occur with normal blood pressure and involves other regions than the posterior brain. The second hypothesis postulates evolving hypertension leads to cerebral vasoconstriction causing hypoperfusion and ischemia with subsequent endothelial injury, capillary leakage \&oedema. ${ }^{7}$ Endothelial dysfunction in systemic diseases is due to immune mediated injury. Excessive cytokines induce astrocytes to make more vascular endothelial growth factor (VEGF), which weakens endothelial cells junctions and disturbs the integrity of blood brain barrier. Inflammatory cytokines like endothelin- 1 cause vasoconstriction. So a composite effect of endothelial injury, leaky tight junctions, leukocyte passage \& vasoconstriction leads to brain oedema. $8,9,10$ This theory explains the often seen watershed pattern of distribution, the ischemia and infarcts in a subset of cases, the petechial haemorrhages, transudation of fluid and large vessel vasospasm that has been encountered on catheter angiography and MR angiography(MRA). ${ }^{11}$

Conditions most commonly associated with PRES

1. Hypertension, Infection/Sepsis

2. Preeclampsia/Eclampsia, Autoimmune diseases

3. Stem cell \& solid organ transplantation

4. Blood transfusion

5. Toxic Agents/Drugs-

a. Cytotoxic Agents: Cisplatin, Carboplatin, Bevacizumab, Sunitinib, IFN- $\alpha$ (Interferon- alpha), IL- 2 (Interleukin), Rituximab, Infliximab, intravenous immunoglobulin (IVIG)

b. Immunosuppressive Drugs: Cyclosporine, Tacrolimus (FK 506), Steroids.

c. Other Agents: Erythropoietin, Intravenous contrast agents.

6. Other Diseases: Sickle cell disease, Guillain- Barré syndrome (GBS), tumour lysis syndrome, porphyria cutanea tarda, pheochromocytoma and Cushing syndrome. 12

Diverse renal parenchymal diseases have been encountered in association with PRES like Lupus nephritis, Focal segmental glomerulonephritis (FSGS), Membranoproliferative glomerulonephritis (MPGN), Post streptococcal glomerulonephritis (PSGN), renovascular hypertension, Henoch- Schonlein purpura(HSP) etc. The pathophysiologic link between these entities and PRES vary from endothelial damage and activation, hypertension, systemic inflammation, cytotoxicity of immunosuppressive therapy and infections to autoimmune processes .10 Our case on biopsy had no glomerular pathology, rather tubulointerstitial inflammation in it. Tubulo- interstitial diseases represent a group of heterogenous disorders with varied aetiology, pathogenesis and clinical course. So the exact cause of TIN and its role in causing PRES could not be ascertained in our case.
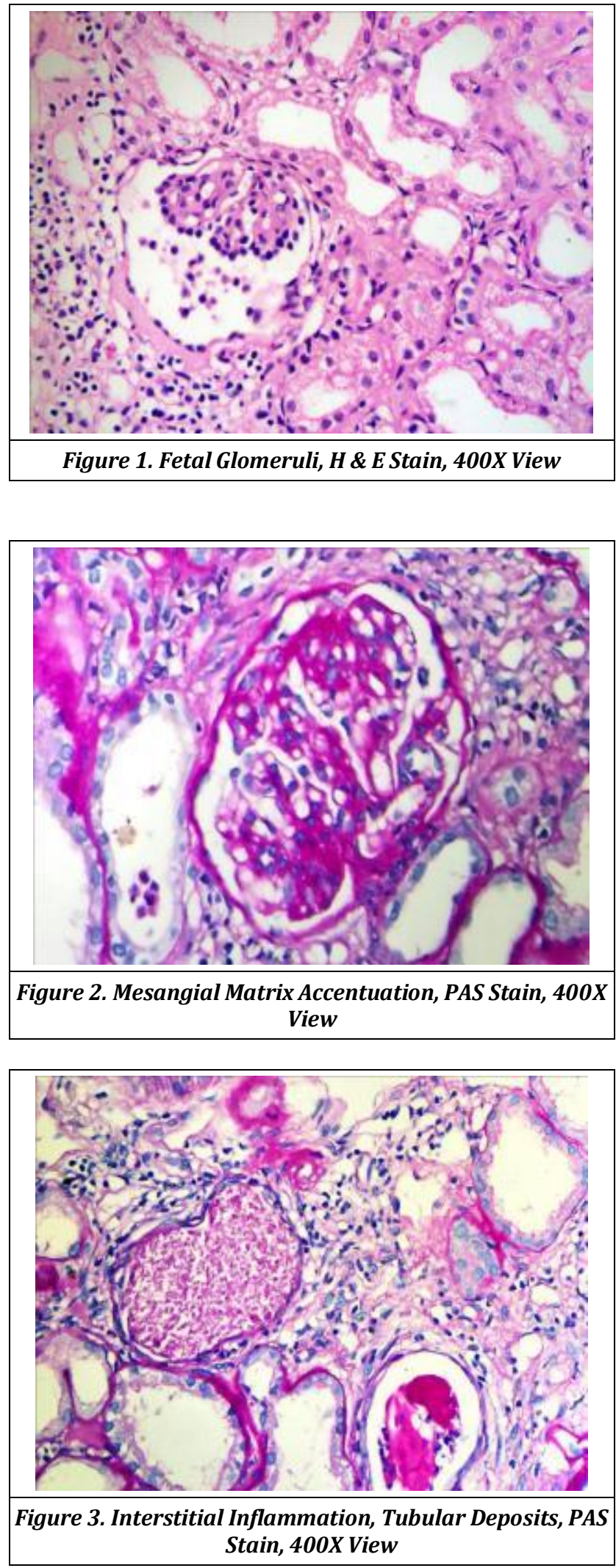

\section{REVIEW OF LITERATURE}

\section{Epidemiology}

The neurological changes seen in patients with renal disease and hypertension was first recognized in 1928 \& termed hypertensive encephalopathy. 4 In 1984 the aetiology was established to be cerebral oedema due to disruption of the blood- brain barrier. ${ }^{5}$ The exact incidence of PRES is unclear. PRES has been reported in patients with wide age range (4 to 90 years) with female predominance. This condition is also 
known as reversible posterior leukoencephalopathy syndrome, reversible posterior cerebral oedema syndrome and reversible occipital parietal encephalopathy. Many patients with PRES have severe comorbidities such as bone marrow or organ transplantation etc. ${ }^{6}$ The acronym PRES gained popularity in year 2000 due to an enhanced ability of FLAIR imaging to detect subtle \& minute peripheral lesions compared to conventional MRI. ${ }^{2}$

\section{Clinical Features}

The typical features of PRES include consciousness impairment, seizure, focal neurological deficit, visual acuity disturbances, headaches, nausea and vomiting. Consciousness impairment may vary from confusion, somnolence and lethargy to encephalopathy or coma. Seizures are tonic- clonic in nature. $2,3,13$ The most common clinical features are seizure $(60-75 \%), 3,14,15$ headache (50\%),16,17 encephalopathy (50$80 \%),{ }^{18,19}$ visual disturbances (33\%),6,14 focal neurological deficit(10-15\%) ${ }^{14,20}$ and status epilepticus (5-15\%) ${ }^{3,14}$ Focal neurological deficit, such as cortical blindness, cerebellar syndrome or hemiparesis may lead to coma. ${ }^{21,22}$

\section{Approach to Diagnosis}

In the presence of acute neurological symptoms as mentioned above and presence of risk factors (e.g.: Hypertension, renal failure, immunosuppressive or chemotherapy, autoimmune disorders or eclampsia), if brain imaging studies show presence of bilateral vasogenic oedema; excluding the other causes first, PRES should be considered as the cause. Combination of clinical manifestations with radiological evidence establishes the diagnosis. However imaging findings may vary depending on the severity of disease. So, awareness regarding variable imaging findings is helpful for diagnosis. In suspicious cases, after initiation of therapy, improved clinical and radiological outcomes, confirms the diagnosis. As till now no consensus guidelines for validation of PRES diagnosis exists, so clinical judgment is considered crucial. ${ }^{3,6,12}$

\section{Radiological Characteristics}

Magnetic resonance imaging (MRI) imaging in PRES typically reveals T2 and FLAIR symmetric reversible hyper intensities in a parieto- occipital region. Computed tomography (CT) scan of the brain shows nonspecific low attenuation lesions.2,8,23 Complications such as cerebral ischemia and cerebral haemorrhage also can be detected radiologically. ${ }^{12}$

\section{Aberrant Presentations}

The clinicians should be acquainted with few non- typical radiological findings. In some scenarios, the lesions may involve basal ganglia (14\%), brain stem $(13 \%)$ or the deep white matter, especially the corpus callosum (10\%). Involvement may be limited to one side of brain only. In some cases, enhancement on T1- weighted images seen in MRI with gadolinium injection. Haemorrhage may develop (5- 30\%) which can intra- parenchymal or sulcal subarachnoid in location. 22,24

\section{Prognosis}

PRES is known to have favourable outcome as most patients recover completely within one week, though some may take longer time. Mortality is reported to be 3-6\%. Hyperglycaemia and time of control of the causative agent are independent poor prognostic factors. 16,25 Complications arise due to intracranial haemorrhage, brainstem compression, acute hydrocephalus and raised intracranial pressure. 26, 27 10-20\% show unremitting hemiparesis, seizures, head reeling \& impaired vision. 28 Women with complicated pregnancies report more protracted cognitive issues. ${ }^{29}$ Recurrence is seen in $5-10 \%$ of cases and associated with uncontrolled hypertension. 30

\section{PRES in Paediatric Age Group}

The clinical and radiological features of PRES in children are found to be similar to that of adults as per few available small case studies and case reports. Acute leukaemia, glomerulonephritis, haemolytic uraemic syndrome (HUS), Henoch- Schonlein purpura (HSP), use of cytotoxic drugs are closely associated with PRES in children. Incidence ranges from $0.4 \%$ to $5.2 \% .^{2,31}$ Though hypertension is common in paediatric patients, the mean blood pressure at the onset of symptoms is lower than adults. ${ }^{32}$ Seizures are the most common presenting symptom (90\%) followed by encephalopathy, visual disturbances, headache and focal neurological deficits. $2,33,34$

Failure to recognize and treat PRES can have devastating consequences. In the presence of neurological symptoms, brain MRI rapidly confirms the diagnostic suspicion, thus directing work- up and treatment.

\section{DISCUSSION OF MANAGEMENT}

The treatment strategy relates to early diagnosis \& general measures to correct the underlying cause of PRES. Patients may need immediate correction of blood pressure or seizure, withdrawal of offending drugs or agents, dialysis or other interventions. 6,12

\section{FINAL DIAGNOSIS}

Tubulointerstitial Nephritis with Mild Mesangial Sclerosis in Association with PRES.

\section{REFERENCES}

[1] Polonara G, Mattioli G, D'ercoli E, et al. Posterior reversible encephalopathy syndrome in children: report of 10 cases.European Society of Radiology2011;C- 1360:1- 4.

[2] Raj S, Overby P, Erdfarb A, et al. Posterior reversible encephalopathy syndrome: incidence and associated factors in a pediatric critical care population. Pediatric Neurology 2013;49(5):335- 9.

[3] Fugate JE, Rabinstein AA. Posterior reversible encephalopathy syndrome: clinical and radiological manifestations, pathophysiology and outstanding questions. Lancet Neurology 2015;14(9):914- 25.

[4] Oppenheimer BS, Fishberg AM. Hypertensive encephalopathy. Archives of Internal Medicine(Chicago) 1928;41:264- 78.

[5] Tamaki K, Sadoshima S, Baumbach GL, et al. Evidence that disruption of the blood brain barrier precedes reduction in cerebral blood flow in hypertensive encephalopathy. Hypertension 1984;6(2 Pt 2):I75- 81.

[6] Legriel S, Pico F, Azoulay E. Understanding posterior reversible encephalopathy syndrome. Annual Update in Intensive Care and Emergency Medicine. Springer 2011: p. 631- 53. 
[7] Hinchey J, Chaves C, Appignani B, et al. A reversible posterior leukoencephalopathy syndrome. New England Journal of Medicine 1996;334(8):494- 500.

[8] Hagea P, Kseiba C, Hmaimessb G, et al. Recurrent posterior reversible encephalopathy syndrome with cerebellar involvement leading to acute hydrocephalus. Clinical Neurology and Neurosurgery 2018;172:120- 3.

[9] Bartynski WS. Posterior reversible encephalopathy syndrome, part 2: controversies surrounding pathophysiology of vasogenicedema. American Journal of Neuroradiology 2008;29(6):1043- 9.

[10] Rykken JB, McKinney AM. Posterior reversible encephalopathy syndrome. Seminar in Ultrasound CT \& MRI 2014;35(2):118- 35.

[11] Casey SO, Sampaio RC, Michel E, et al. Posterior reversible encephalopathy syndrome: utility of fluidattenuated inversion recovery MR imaging in the detection of cortical and subcortical lesions. American Journal of Neuroradiology 2000;21(7):1199- 206.

[12] Arzanian MT, Shamsian BS, Karimzadeh P, et al. Posterior reversible encephalopathy syndrome in pediatric hematologic- oncologic disease: literature review and case presentation. Iranian Journal of Child Neurology 2014;8(2):1- 10.

[13] Kastrup O, Gerwig M, Frings M, et al. Posterior reversible encephalopathy syndrome (PRES): electroencephalographic findings and seizure patterns. Journal of Neurology 2012;259(7):1383- 9.

[14] Burnett MM, Hess CP, Roberts JP, et al. Presentation of reversible posterior leukoencephalopathy syndrome in patients on calcineurin inhibitors. Clinical Neurology \& Neurosurgery 2010;112(10):886- 91.

[15] Liman TG, Bohner G, Heuschmann PU, et al. The clinical and radiological spectrum of posterior reversible encephalopathy syndrome: the retrospective Berlin PRES study.J Neurol2012;259(1):155- 64.

[16] Tlemsani C, Mir O, Boudou- Rouquette P, et al. Posterior reversible encephalopathy syndrome induced by antiVEGF agents.Target Oncology 2011;6(4):253- 8.

[17] Li Y, Gor D, Walicki D, et al. Spectrum and potential pathogenesis of reversible posterior leukoencephalopathy syndrome.Journal of Stroke \& Cerebrovascular Disease 2012;21(8):873- 82.

[18] Brewer J, Owens MY, Wallace K, et al. Posterior reversible encephalopathy syndrome in 46 of 47 patients with eclampsia.American Journal of Obstetrics \&Gynecology2013;208(6):468.e1-e6.

[19] Liman TG, Bohner G, Heuschmann PU, et al. The clinical and radiological spectrum of posterior reversible encephalopathy syndrome: the retrospective Berlin PRES study. J Neurol2012;259(1):155- 64.

[20] Cruz RJ Jr, DiMartini A, Akhavanheidari M, et al. Posterior reversible encephalopathy syndrome in liver transplant patients:clinical presentation, risk factors and initial management.Am J Transplant 2012;12(8):2228- 36.
[21] Lee VH, Wijdicks EF, Manno EM, et al. Clinical spectrum of reversible posterior leukoencephalopathy syndrome.Arch Neurol 2008;65(2):205- 10.

[22] Hugonnet E, Da Ines D, Boby $\mathrm{H}$, et al. Posterior reversible encephalopathy syndrome(PRES): features on CT and MR imaging. Diagnostic and Interventional Imaging 2013;94(1):45- 52.

[23] Chen Z, Shen GQ, Lerner A, et al. Immune system activation in the pathogenesis of posterior reversible encephalopathy syndrome. Brain Res Bull 2017;131:93- 9 .

[24] Hamilton BE, Nesbit GM. CSF enhancement in posterior reversible encephalopathy syndrome. AJNR Am J Neuroradiol 2008;29(3):456- 7.

[25] Legriel S, Schraub O, Azoulay E, et al. Determinants of recovery from severe posterior reversible encephalopathy syndrome.PLoS One 2012;7(9):e44534.

[26] Grossbach AJ, Abel TJ, Hodis B, et al. Hypertensive posterior reversible encephalopathy syndrome causing posterior fossa edema and hydrocephalus. Journal of Clinical Neuroscience 2014;21(2):207- 11.

[27] Lee SY, Dinesh SK, Thomas J. Hypertension- induced reversible posterior leukoencephalopathy syndrome causing obstructive hydrocephalus. Journal of Clinical Neuroscience 2008;15(4):457- 9.

[28] Striano P, Striano S, Tortora F, et al. Clinical spectrum and critical care management of Posterior Reversible Encephalopathy Syndrome(PRES). Med Science Monit2005;11(11):CR549- 53.

[29] Postma IR, Slager S, Kremer HP, et al.Long- term consequences of the posterior reversible encephalopathy syndrome in eclampsia and preeclampsia:a review of the obstetric and nonobstetric literature.Obstetrics \&GynecologySurv2014;69(5):287- 300.

[30] Li R, Mitchell P, Dowling R, et al. Is hypertension predictive of clinical recurrence in posterior reversible encephalopathy syndrome? Journal of Clinical Neuroscience 2013;20(2):248- 52.

[31] Cordelli DM, Masetti R, Bernardi B, et al. Status epilepticus as a main manifestation of posterior reversible encephalopathy syndrome after pediatric hematopoietic stem cell transplantation. Pediatric Blood Cancer 2012;58(5):785- 90.

[32] Brady KM, Mytar JO, Lee JK, et al. Monitoring cerebral blood flow pressure autoregulation in pediatric patients during cardiac surgery.Stroke 2010;41(9):1957- 62.

[33] De Laat P, TeWinkel ML, Devos AS, et al. Posterior reversible encephalopathy syndrome in childhood cancer. Annals of Oncology 2011;22(2):472- 8.

[34] Siebert E, Spors B, Bohner G, et al. Posterior reversible encephalopathy syndrome in children: radiological and clinical findings - a retrospective analysis of a German tertiary care center. European Journal of Paediatric Neurology 2013;17(2):169- 75. 